CERN-TH/96-326

USC-96/026

hep-th/9612085

\title{
Non-Critical Strings, Del Pezzo Singularities and Seiberg-Witten Curves
}

\author{
W. Lerche ${ }^{a}$, P. Mayr ${ }^{a}$ and N.P. Warner ${ }^{b}$ \\ ${ }^{a}$ Theory Division, CERN, 1211 Geneva 23, Switzerland \\ ${ }^{b}$ Physics Department, U.S.C., University Park, Los Angeles, CA 90089
}

\begin{abstract}
We study limits of four-dimensional type II Calabi-Yau compactifications with vanishing four-cycle singularities, which are dual to $\mathbf{T}^{2}$ compactifications of the six-dimensional non-critical string with $E_{8}$ symmetry. We define proper subsectors of the full string theory, which can be consistently decoupled. In this way we obtain rigid effective theories that have an intrinsically stringy BPS spectrum. Geometrically the moduli spaces correspond to special geometry of certain non-compact Calabi-Yau spaces of an intriguing form. An equivalent description can be given in terms of Seiberg-Witten curves, given by the elliptic simple singularities together with a peculiar choice of meromorphic differentials. We speculate that the moduli spaces describe non-perturbative noncritical string theories.
\end{abstract}

December 1996

USC-96/026

CERN-TH/96-326 


\section{Introduction}

The relation between singularities in supersymmetric string compactifications on Calabi-Yau manifolds and field theories has added much to the understanding of nonperturbative string theory in the last two years. Starting from the interpretation of threefold singularities in terms of known field theories, the direction has veered around in the meantime to the discussion of novel types of theories to interpret various types of geometrical singularities. Indeed one may expect to find in this way a whole zoo of supersymmetric effective theories, and there is no reason why such theories should always be interpretable in terms of conventional field theories.

In particular, new theories have been discovered that involve non-critical strings in various dimensions [1, 2, 3, 4, 5, 6, 0, 8, 9, 10, 11]. These theories are still difficult to access directly and it is easier to study them after further compactification. For example, new interacting fixed points of five-dimensional gauge theories [12] have been related [13, 14] to $S^{1}$ compactifications of the non-critical string with global $E_{8}$ symmetry in six dimensions. It is interesting to further compactify this theory on another circle, and to investigate the properties of the resulting $d=4 N=2$ supersymmetric theory.

In this note we consider certain limits of four-dimensional Calabi-Yau compactifications of type II strings, which are dual to compactifications of the six-dimensional noncritical $E_{8}$ string on $\mathbf{T}^{2}$. Aspects of the resulting four-dimensional theory have been discussed previously in [8], and more recently, while we were completing this paper, in [15]. However, we will study different aspects of this theory, and, in particular, our focus will be on a quite different, more stringy type of moduli space. More specifically, we will obtain some new insights in this moduli space by making use of the special geometry of certain Calabi-Yau manifolds.

Recall that the non-critical string with global $E_{8}$ symmetry arises in six dimensional Calabi-Yau compactifications of F-theory, when a del Pezzo 4-cycle $\mathbf{B}_{n}$ shrinks to zero size [16, 团. At present, no simple representation of this six-dimensional theory in its own is known, and its existence is based on a collection of evidence rather than on a firm proof. If the six-dimensional string theory really exists, its effective theory should show up as a "closed subsector" of the full type II compactification. Specifically, one should be able to separate off a sub-sector of the BPS states of the full type II theory, which corresponds to

$1 \mathbf{B}_{n}$ denotes the del Pezzo surface obtained from blowing up $\mathbf{P}^{2}$ at $0<n \leq 9$ points. For more details we refer to [14]. 
the embedded six-dimensional theory. This sub-sector should be universal and should not depend on the details of the chosen embedding three-fold.

We indeed find such a sub-theory, which we associate to a certain non-compact CalabiYau manifold as the underlying geometrical object. This structure might hint at a more fundamental formulation of the theory. A peculiar feature of this sub-theory is that it involves the large complex structure limit of the three-fold and thus is inherently stringy.

In section 2 we define consistent sub-moduli spaces of the special geometry describing the effective type II theory, in terms of monodromy and intersection properties. In section 3 we investigate two different sub-moduli spaces related to the tension of the compactified non-critical string, for a specific embedding three-fold, and discuss their physical properties.

In the rest of the paper, we then concentrate only on one of these theories, namely on the one whose moduli space intersects the large complex structure limit. This moduli space corresponds, essentially, to the non-critical string tension. In section 4, we identify the underlying universal geometry in terms of certain Landau-Ginzburg potentials, which involve a coupling of a $x^{-n}$ potential to the local geometry of del Pezzo singularities. The geometrical periods on the associated non-compact Calabi-Yau space turn out to be closely related to those of certain elliptic curves. Specifically, we show in section 5 that the local three-fold geometry can be captured by Seiberg-Witten curves with a particular choice of meromorphic differentials. We then explicitly evaluate in section 6 the periods near the singular points in the moduli space. We verify that the theory is conformal at the origin of the moduli space, and reproduce from the SW formulation of the theory its unusual stringy behaviour near infinity. Finally, in section 7 we conclude with some more speculative remarks.

\section{Definition of the moduli space}

We want to extract the physics of the compactified six-dimensional non-critical $E_{8}$ string (henceforth referred to as "NCS") from the type II moduli space. For this, it will be important to have a clear distinction between the degrees of freedom that are part of the NCS theory, and those that are "superfluous" states of the type II theory. The states of the NCS will have a universal description that is independent of the particular CalabiYau manifold $\mathbf{X}$, whereas the superfluous states will depend upon non-universal, global properties of $\mathbf{X}$.

Near a singular locus in the Calabi-Yau moduli space $\mathcal{M}(\mathbf{X})$, the theory is dominated by a subset $\mathcal{H}$ of the BPS states of the full theory, with a typical mass scale $\Lambda$ much 
smaller than that of the rest of the theory. An important characteristic of the subset $\mathcal{H}$ is the behaviour of the theory away from the singularity. There are, roughly speaking, two possibilities: either the subset of states $\mathcal{H}$ does not extend to a globally well-defined theory away from the particular scaling limit, so that it has to be coupled to (a larger subset of) the rest of the theory; this is, for example, what happens with asymptotically non-free field theories. Or, more interestingly, the subset $\mathcal{H}$ itself provides a globally well-defined theory also away from the singularity, as in SYM theories with asymptotically free spectrum [17].

The effective abelian $N=2$ gauge theory in four dimensions is described by the holomorphic prepotential $\mathcal{F}$ of special geometry, which itself is determined by the period integrals of $\mathbf{X}$. The question of whether the truncated theory obtained in some scaling limit is consistent and has a global extension, translates to the question whether we can find a proper sub-moduli space $\mathcal{M}$ of the full moduli space $\mathcal{M}(\mathbf{X})$. This suggests a general definition of "consistent sub-theories" embedded in string theory, in terms of closed sub-monodromy problems. That is, the full compactified string theory is governed by a monodromy group $G$, and we look for a subgroup $H \subset G$ that acts reducibly on the full set of BPS states and closes on the subset $\mathcal{H}$ of states that are relevant near the given singularity. If such a subgroup exists, we can consistently throw away the remaining sectors of the theory and define a new theory on the moduli space $\mathcal{M}_{H} \subset \mathcal{M}(\mathbf{X})$. One expects that these monodromy data can be associated to a geometrical object, like the Riemann surface in SYM theories.

In fact, since it is known that the generic monodromy group of a Calabi-Yau compactification is generated by the monodromy on a generic hyperplane in the (properly resolved) moduli space, we could start a systematic search for such theories by classifying the (intersections of) non-generic hyperplanes in $\mathcal{M}(\mathbf{X})$. Instead we will restrict ourselves to sub-problems which involve a large complex structure point. In this case, it is simple to find a necessary condition for the existence of a subgroup of the monodromy group. At a large complex structure point with maximal unipotent monodromy, the Calabi-Yau periods, when written in special coordinates $t_{i}$, have the asymptotic form:

$$
\Pi \sim\left(1, \quad t_{k}, \quad \frac{1}{2} c_{i j k} t^{i} t^{j}+\ldots, \quad-\frac{1}{6} c_{i j k} t^{i} t^{j} t^{k}+\ldots\right),
$$

where the $c_{i j k}$ are the triple intersections on $\mathbf{X}$ and the ellipses denote subleading terms in the limit $t_{i} \rightarrow \infty$ (which behave in a similar way). It follows that a necessary condition for the existence of a subset $S(H)$ of periods closed under the monodromy at infinity is that their intersection form with the rest of the periods vanishes. Geometrically this means that 
we can find a basis2 2 of divisors in $H_{4}(\mathbf{X}, \mathbf{Z})$, such that the subset $\mathcal{S}(H)$ does not intersect with divisors that are not in $\mathcal{S}(H)$.

The above implies a rather interesting property of this kind of sub-monodromy problems: in contrast to the embeddings of SYM theories considered previously [18, 19, these theories inherit the instanton expansion - that is, the Gromov-Witten invariants - from the Calabi-Yau manifold. In the special sub-monodromy problem that we will consider below, this is related to the fact that the underlying geometry is that of a non-compact Calabi-Yau space of a very special form. This suggests an alternative interpretation of the splitting condition on the intersection form, namely in terms of the existence of a well-defined, possibly non-compact Calabi-Yau space that captures the relevant part of the geometry.

There is also the possibility of treating some of the Calabi-Yau moduli as background fields rather than as dynamical quantities. In this case the restriction on the intersections has to be imposed only on the periods treated as moduli. An obvious example are mass parameters in an effective field theory, which originate as gauge masses in the full string theory.

\section{The NCS as a sub-monodromy problem}

We now apply the above considerations to the elliptically fibered three-fold $\mathbf{X}_{\mathbf{F}_{1}}$ with base $\mathbf{F}_{1}$, which provides the simplest embedding for the kind of theory we want to study. Note, however, that the subsector corresponding to the NCS depends only on the local geometry, and that the results we will obtain turn out to be independent of the three-fold that we choose as an embedding.

F-theory on $\mathbf{X}_{\mathbf{F}_{1}}$ is dual to the heterotic string on $K 3$ with instanton embedding $\left(n_{1}=11, n_{2}=13\right)$ in the two $E_{8}$ factors, respectively. The vanishing 4-cycle singularity of the Calabi-Yau compactification maps to the strong coupling singularity of the heterotic dual. At this singularity, a non-critical string becomes tensionless. It is the same string [4,16] as the one stretched between the fivebrane and the ninebrane [3] in M-theory on $\mathbf{S}^{1} / \mathbf{Z}_{2} \times K 3$, for a reason explained in 9 .

The four-dimensional $N=2$ supersymmetric theory, obtained by compactification on a further $\mathbf{T}^{2}$, is dual to the type IIA string on $\mathbf{X}_{\mathbf{F}_{1}}$. There are two Calabi-Yau phases, which are related by a flop; we denote them by Phase $I$ and and Phase $I I$, respectively.

2 In some cases it may be useful to relax the condition that the basis is integral, in particular if the non-integrality is related to elements not in $S(H)$. 
The $h^{1,1}=3$ vector multiplet fields $t_{E}, t_{F}$ and $t_{B}$ parametrize in Phase $I$ the volume of a curve in the fiber and the two classes of $\mathbf{F}_{1}$, respectively. The base of $\mathbf{F}_{1}$, with volume $t_{B}$, is also the base of a $\mathrm{K} 3$ fibration and is therefore related to the heterotic dilaton. Figure 1 shows a real slice $i t_{i} \in \mathbb{R}$ through $\mathcal{M}\left(\mathbf{X}_{\mathbf{F}_{1}}\right)$, and displays the singularities associated to the flop and the vanishing 4-cycle, respectively.

The leading pieces of the prepotentials $\mathcal{F}_{I}, \mathcal{F}_{I I}$, which in particular summarize the intersection properties of divisors in $\mathbf{X}_{\mathbf{F}_{1}}$, are given in Appendix A. A characteristic split of the intersection form, indicating a possible closed sub-monodromy problem which intersects the large complex structure point, can be observed in Phase $I I$. More precisely, in a basis, where the scalar fields in the vector multiplets are given by $t_{S} \equiv t_{E}+t_{B}, t_{F}^{\prime} \equiv t_{F}+t_{B}$, and $t_{E}$, there are three periods which depend classically only on one of the three fields.

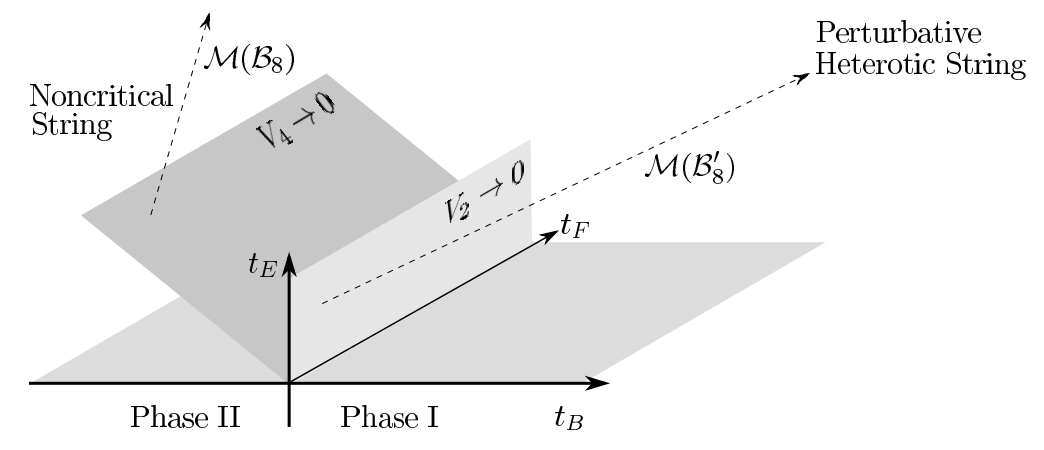

Fig.1: Real slice through the Kähler moduli space of $\mathbf{X}_{\mathbf{F}_{1}}$. The planes $V_{2} \rightarrow 0$ and $V_{4} \rightarrow 0$ denote the faces of the Kähler cone where a 2-cycle (flop) and a 4-cycle (del Pezzo) shrink, respectively. We refer to the phases with $t_{B}>0\left(t_{B}<0\right)$ as Phase $I$ (II). We have also sketched the moduli spaces of the effective theories $\mathcal{B}_{8}, \mathcal{B}_{8}^{\prime}$ of the NCS that we will consider below. Far out at infinity in Phase $I$ is the region of the perturbative heterotic string. On the other hand, Phase II is not a $K 3$ fibration and thus not related to the perturbative heterotic string.

Continuation beyond the flop reveals that this splitting property is no longer present in Phase I. For a globally consistent definition of this system beyond the flop, we have to include one further period as a background parameter, namely the volume of the elliptic fiber $t_{E}$. So depending on whether we include $t_{E}$, or not, we have two subsets of periods that define appropriate sub-monodromy problems:

$$
\tilde{\Pi}_{i}=\left(1, t_{S}, \partial_{t_{S}} \mathcal{F}\right) \quad \tilde{\Pi}_{i}^{\prime}=\left(1, t_{S}, \partial_{t_{S}} \mathcal{F}, t_{E}\right) .
$$

In the following we will mainly focus on the three periods $\tilde{\Pi}_{i}$, which correspond to $0,2,4$ cycles in the three-fold, respectively; the 4-cycle is the del Pezzo $\mathbf{B}_{8}$ in question. It is 
gratifying to observe that the single Kähler coordinate that is treated as a modulus in $\tilde{\Pi}_{i}$, namely $t_{S}$, is precisely the one which parametrizes the volume of the 4-cycle.3

For a complete definition of the theory we have still to choose values for the other moduli, at which we vary $t_{S}$. In fact, different choices will lead to rather different physics. In particular, the BPS spectrum at the singularities of the moduli space $\mathcal{M}_{S}$ will depend which set of the Calabi-Yau singularities is hit by this moduli space.

\section{Effective theories of the NCS}

To relate the physics of the embedded theory, defined by the periods (2.2), to the NCS, we have to find the relation between the Calabi-Yau moduli and the fields of the effective theory of the NCS. This can be done by extending the five-dimensional analysis of [6,7]. In the five-dimensional compactification, the radius $R_{6}$ of $\mathbf{S}^{1}$ is inversely proportional to the size of the elliptic fiber, $t_{E}$. Moreover, the tension of the magnetically charged string is proportional to the volume $V_{4}$ of the 4 -cycle $\mathbf{B}_{n}$. This volume can be parametrized by the Kähler coordinate $t_{S}$, which measures the volume of 2-cycles within $\mathbf{B}_{n}$. More precisely one has: $V_{4} \sim t_{S}^{2}$.

For a further $\mathbf{S}^{1}$ compactification of the five-dimensional theory to four dimensions, the relation between the coordinates is then given by $t_{i}^{(4)}=i R_{5} t_{i}^{(5)}$ 20]. This leads to the approximate identifications:

$$
t_{S}=: \tilde{\phi}=i \phi R_{6} R_{5}, \quad t_{E}=: \mathcal{U}=i \frac{R_{5}}{R_{6}}, \quad t_{F}^{\prime}=: \mathcal{T}=i \phi_{F} R_{6} R_{5}+\mathcal{O}\left(R_{6}^{0}\right)
$$

where $\phi\left(\phi_{F}\right)$ is the tension of the non-critical (fundamental) string 1 .

The precise identifications, which take a non-diagonal metric of the torus and the $B$-field into account, can be inferred from the map to the moduli of the heterotic dual in the weakly coupled regime. These moduli are the dilaton $S$, and the Kähler and complex structure of the torus, i.e. $T=B+i R_{5} R_{6}, U=e^{i \alpha} R_{5} / R_{6}$. Comparing the prepotential $\mathcal{F}_{I}$ (A.1) of the K3 fibered Phase $I$ with that of the heterotic string, where $\mathcal{F}_{\text {het }}=S T U+$

3 More generally, one could consider higher dimensional moduli spaces by including additional homology classes of the del Pezzo surface, corresponding to non-zero Wilson lines of the heterotic theory.

4 We set $\phi_{F}=1$ if not denoted otherwise. 
$\mathcal{O}\left(e^{2 \pi i S}\right)$, one obtains $t_{F}=T-U, t_{B}=S+a T+b U, t_{E}=U$ (here we have assumed that $\operatorname{Im}(T)>\operatorname{Im}(U))$.

As mentioned before, the effective $N=2 U(1)$ theory is determined after fixing the values of $\mathcal{U}$ and $\mathcal{T}$. The theory obtained in this way can be suitably characterized by the surviving BPS spectrum. For this, let us first reconsider the five-dimensional theory, which contains the relevant features of the four-dimensional case.

Point-like BPS states in five dimensions have masses determined by the central charge 21

$$
Z=\sum_{i} n_{i} t_{i}^{(5)}=\frac{1}{\mathcal{V}_{5}^{1 / 3}}\left(l \cdot \phi R_{6}+p \cdot \frac{1}{R_{6}}+m \cdot\left(\phi R_{6}-2 \frac{1}{R_{6}}+R_{6}\right)\right)
$$

where the $t_{i}^{(5)}$ are subject to the constraint $\mathcal{V}_{5}\left(t_{i}^{(5)}\right)=1$, with $\mathcal{V}_{5}(v)=c_{i j k} v_{i} v_{j} v_{k}$ and $c_{i j k}$ the intersection numbers 20].

Phase I (II) corresponds to the region $\phi R_{6}>\frac{1}{R_{6}}\left(\phi R_{6}<\frac{1}{R_{6}}\right)$. As shown in Fig. 1, far out in the Kähler cone of Phase $\mathrm{I}$ is the regime of the perturbative heterotic theory, and the light states are the fundamental string windings and momenta with quantum numbers $q \equiv(l, p, m)=(l, p,-l)$. As one increases the tension $\phi$, one hits the boundary between phases $I$ and $I I$. Associated to the vanishing 2-cycle is a massless hypermultiplet [4] with winding/momentum $q=(l, p, m)=(1,-1,0)$ [7]. In fact, in this limit there is also a net number of 480 fundamental string momentum modes that are equally relevant. They correspond to rational curves in the fiber and their number has a simple interpretation: T-duality in the fiber transforms them to 0-branes, whose moduli space is the Calabi-Yau $\mathbf{X}_{\mathbf{F}_{1}}$ itself - with Euler number $\chi=-480$.

Geometrically, the 4-cycle which is shrunk at the singularity $\tilde{\phi}=0$ is still of type $\mathbf{B}_{9}$ in Phase $I$ and becomes type $\mathbf{B}_{8}$ in Phase $I I$. It is thus clear that there will be two different classes of effective theories, whose moduli spaces intersect at the singularity $\tilde{\phi}=0$ : for any finite value of $\mathcal{U}$, increasing the string tension $\phi$ will finally let us hit the wall $\phi R_{6}=\frac{1}{R_{6}}$, where an additional hypermultiplet becomes massless and changes the asymptotic behaviour of the theory. In fact, the same will happen when starting from the $\mathbf{B}_{n}$ type of singularity, where one hits successively the walls corresponding to massless hypermultiplets, as is clear from the alternative description in terms of D4-branes [13] [14]. In physical terms one ends up with a matter content $N_{f}=8$ of an $S U(2)$ gauge theory, whose Coulomb branch is parametrized by $\tilde{\phi}$. This theory has a trivial dependence on the

5 The constants $a$ and $b$ remain undetermined in perturbation theory. 
Coulomb modulus, that is, a constant effective coupling [12]. We will refer to this kind of theory, which starts from the $\mathbf{B}_{n}$ singularity and ends up in the $\mathbf{B}_{9}$ phase, as $\mathcal{B}_{n}^{\prime}$.

On the other hand, if one wants to keep the theory in the phase with a 4-cycle of type $\mathbf{B}_{8}$, one has to send $\mathcal{U}$ to infinity first, such that the wall of the flop is shifted away by an infinite distance. We will refer to this type of sub-theory as $\mathcal{B}_{n}$. It is this kind of theories that we will mainly focus on in this paper.

The situation is similar after further compactification to four dimensions: for any finite value of the modulus $\mathcal{U}$, the four-dimensional theory reaches phase $I$ for large values of the string tension, and transmutes to the $\mathcal{B}_{9}$ type of theory. We will denote the resulting theories by $\mathcal{B}_{n}$ and $\mathcal{B}_{n}^{\prime}$ as well. The locations of the moduli spaces of the theories $\mathcal{B}_{8}$ and $\mathcal{B}_{8}^{\prime}$ in $\mathcal{M}(\mathbf{X})$ are indicated in Fig. 1.

\section{The effective theory $\mathcal{B}_{8}^{\prime}$}

This is the theory with a trivial asymptotic behaviour. The periods dual to $\tilde{\phi}$, obtained from (A.1), are:

$$
\begin{aligned}
\text { Phase } I: & \tilde{\phi}_{D}=-\frac{1}{2} \mathcal{U}^{2}+\tilde{\phi} \mathcal{U}-\frac{1}{2} \mathcal{U}-\frac{1}{2}+\partial_{\tilde{\phi}} \mathcal{F}_{I}^{\text {inst }}, \\
\text { Phase } I I: & \tilde{\phi}_{D}=\frac{1}{2} \tilde{\phi}^{2}-\frac{1}{2} \tilde{\phi}-\frac{5}{12}+\partial_{\tilde{\phi}} \mathcal{F}_{I}^{\text {inst }} .
\end{aligned}
$$

Restricting to the quadratic terms in (3.3) one recovers the five-dimensional expressions [13,14]. The four-dimensional BPS mass formula reads in special coordinates [22]:

$$
Z=e^{K / 2}\left(1 \cdot n_{0}+t_{i} \cdot n_{i}-\mathcal{F}_{0} \cdot m_{0}-\mathcal{F}_{i} \cdot m_{i}\right),
$$

where $K$ is the Kähler potential, $\mathcal{F}_{i}=\partial_{t_{i}} \mathcal{F}$ and $\mathcal{F}_{0}=2 \mathcal{F}-t_{i} \mathcal{F}_{i}$. Moreover, $n_{A}$ and $m_{A}$, $A=0 \ldots 3$, are integer charges. Restricting to the periods of the second sub-problem in (2.2), $\tilde{\Pi}_{i}^{\prime}=\left(1, t_{S}, \partial_{t_{S}} \mathcal{F}, t_{E}\right) \equiv\left(1, \tilde{\phi}, \tilde{\phi}_{D}, \mathcal{U}\right)$, is justified if all other periods are much larger. To achieve this, we have still to decouple part of the type IIA string states. The only modulus that involves explicitly the fundamental string tension $\phi_{F}$, is $\mathcal{T}$ :

$$
\operatorname{Im}(\mathcal{T})=\phi R_{5} R_{6} \sin \alpha-2 R_{5} / R_{6} \sin \alpha+\phi_{F} R_{5} R_{6} \sin \alpha .
$$

Thus, in order to decouple the fundamental string states, we send $\phi_{F} \rightarrow \infty$ and obtain:

$$
Z=R_{5} e^{K / 2}\left(\frac{n_{0}}{R_{5}}+n_{2} \phi R_{6}+\frac{n_{3}}{R_{6}}-m_{2} \tilde{\phi}_{D}\right) .
$$

6 These theories have been considered in [15]. 
From $(3.5)$ and $\tilde{\phi}_{D} \sim \tilde{\phi} R_{5}+\mathcal{O}\left(\tilde{\phi}^{0}\right)$ we see that the states with masses $m=|Z|$ can be interpreted as the winding and momentum states of the NCS on the torus. Furthermore, as can be seen from (3.3), the monodromy at infinity $\tilde{\phi} \rightarrow \tilde{\phi}+1$ acts on the periods as

$$
\left(1, \tilde{\phi}, \tilde{\phi}_{D}, \mathcal{U}\right) \quad \rightarrow \quad\left(1, \tilde{\phi}+1, \tilde{\phi}_{D}+\mathcal{U}, \mathcal{U}\right)
$$

It acts trivially on the $U(1)$ gauge coupling, $\tau(\tilde{\phi})=\partial_{\tilde{\phi}} \tilde{\phi}_{D}$, as has been observed also in [15].

The effective theory $\mathcal{B}_{8}$

The behaviour of the $\mathcal{B}_{8}$ theory turns out to be rather different. As mentioned before, if we send $\mathcal{U} \rightarrow \infty$ first, we stay always in Phase $I I$. There is no need to include the period $\mathcal{U}$, because the charged states associated to it have become infinitely massive.

The gauge coupling behaves like $\tau(\tilde{\phi}) \sim \tilde{\phi}$, as is familiar from the large complex structure limit; it is typical for a decompactification limit. From (3.3) and (3.4) one finds that for large $\tilde{\phi}$, the BPS spectrum contains states with masses

$$
m \sim \quad \tilde{\phi}, \quad 1, \frac{1}{\tilde{\phi}}
$$

up to multiplication with a common mass scale. In particular, since $\tilde{\phi}=b+i R_{5} R_{6} \phi$, these masses depend only on the $b$ field and the tension times a volume factor, which in turn can be reabsorbed in the tension. This is very much like in a "pure" uncompactified string theory, where there are no relevant geometrical parameters to be varied.

From the type IIA perspective the above states correspond to 4, 2 and 0 brane states, the latter ones being the relevant states for large $\tilde{\phi}$, uncharged under the $U(1)$ gauge symmetry. The states whose mass depends inversely proportional to the volume can be interpreted as Kaluza-Klein modes. The states with masses $\sim \tilde{\phi}, \sim 1$ (up to a prefactor $\tilde{\phi})$ are the ones which become massless at $\tilde{\phi}=0$ (despite quantum corrections). There is one further massless state for a value $1 / 2$ of the $b$ field, namely a $0-4$ brane bound state, which leads to a conifold singularity, as we will see later on.

\section{Gauge couplings in five and four dimensions}

A crucial difference between the five-dimensional and four-dimensional fixed point theories is the value of the $U(1)$ gauge coupling, $\tau=\partial_{\tilde{\phi}} \tilde{\phi}_{D}(\mathrm{cf}$. , (3.3)) at the critical point $\tilde{\phi} \sim \epsilon \rightarrow 0$ : it is infinity in five [12], but should be at a fixed point of the modular group in four dimensions [23]. 
Geometrically, infinite coupling in five dimensions is the statement that if the 2-cycle volume goes to zero as $V_{2}=\tilde{\phi} \sim \epsilon$, the 4 -cycle volume vanishes as $\tilde{\phi}^{2} \sim \epsilon^{2}$. This follows from the quadratic terms in (3.3):

$$
\frac{1}{g_{0}^{2}} \sim \partial_{\tilde{\phi}} \tilde{\phi}_{D} \sim \epsilon
$$

In four dimensions, quantum corrections do not lead to a finite volume for neither the 4-cycle nor the 2-cycle [10], so that cannot be the explanation for the finite value of the coupling constant. However, from (3.7) it is clear that it is enough that the instanton corrections change the scaling from $V_{4} \sim V_{2}^{2}$ to $V_{4} \sim V_{2}$. We will see below in section 6 that this is precisely what happens, that is, $V_{4} \sim \tau V_{2}$ with $\tau_{0}$ the gauge coupling at the fixed point.

This has an interesting consequence which could have been anticipated on physical grounds. As explained in [7], there are states in the four-dimensional type IIA theory with

masses $\sim V_{2}, \sim V_{4}, \sim V_{4}^{\frac{1}{2}}$ that arise from wrapping the D2, D4 and 5 brane, respectively. Would we still have $V_{4} \sim V_{2}^{2}$ as in five dimensions, the most relevant states would be the wrapped D4 branes and this would lead to a theory with mutually local "magnetic" charges. This would be in contradiction with the experience that conformal fixed points always involve states with mutually non-local charges [24]. However, if $V_{4} \sim V_{2}$, the D2 brane state is as relevant as the one from the D4 brane, implying the presence of mutually non-local massless BPS states.

\section{Intrinsic geometrical formulation of the theories $\mathcal{B}_{n}$}

We will now consider in some more detail the four-dimensional theories $\mathcal{B}_{n}$ ( $n=$ $6,7,8)$ as closed sub-theories by themselves. In particular, we will formulate these theories independently of an embedding in a "larger" Calabi-Yau manifold.

Let us first ask about the geometrical object behind the sub-monodromy problem defined and described above. It is a remarkable fact that we can associate Gromov-Witten invariants with a sub-monodromy problem that depends only on a single modulus $t_{S}$ (associated to the volume of 2-cycles in the del Pezzo surface $\mathbf{B}_{n}$ ). One may wonder about why this separation of the del Pezzo surface from the whole Calabi-Yau three-fold works, since the del Pezzo has a non-vanishing first Chern class, $c_{1} \neq 0$; one thus expects a nontrivial coupling to two-dimensional gravity. Recall therefore the description of the conifold singularity in ref. [25]. Although the conifold geometry is locally not Ricci flat, it can be 
described by a Landau-Ginzburg model which combines the description of the $\hat{c}=1$ string in terms of the WZW theory $S U(2)$ at level $k=-3$ [26], with an ALE space with $A_{1}$ singularity:

$$
W_{C F}=\frac{1}{x}+y_{1}^{2}+y_{2}^{2}+y_{3}^{2}+y_{4}^{2}
$$

This Landau-Ginzburg potential is related to a hypersurface $\mathbf{P}^{-2,1,1,1,1}[2]$ and satisfies the Calabi-Yau condition with $\hat{c}=3$. ADE type of generalizations of this Landau-Ginzburg potential, which involve the coupling of $1 / x^{\ell}$ potentials to ALE spaces, have been considered in [27].

In the present case, the local geometry of the singularity is described by the vanishing del Pezzo 4-cycle $\mathbf{B}_{n}$ instead of an ALE singularity. We are thus led to consider the following Landau-Ginzburg potentials:

$$
\begin{aligned}
\mathbf{P}^{3,2,1,1,-1}[6]: & W_{E_{8}}=\frac{1}{x^{6}}+y^{2}+w^{3}+z_{1}^{6}+z_{2}^{6}-\psi x y z_{1} z_{2} w \\
\mathbf{P}^{2,1,1,1,-1}[4]: & W_{E_{7}}=\frac{1}{x^{4}}+y^{2}+w^{4}+z_{1}^{4}+z_{2}^{4}-\psi x y z_{1} z_{2} w \\
\mathbf{P}^{1,1,1,1,-1}[3]: & W_{E_{6}}=\frac{1}{x^{3}}+y^{3}+w^{3}+z_{1}^{3}+z_{2}^{3}-\psi x y z_{1} z_{2} w \\
\mathbf{P}^{1,1,1,1,1,-1}[2,2]: & W_{E_{5}}^{1}=\frac{1}{x^{2}}+z_{1}^{2}+z_{2}^{2}+z_{3}^{2}-\psi x z_{3} z_{4} z_{5} \\
& W_{E_{5}}^{2}=z_{3}^{2}+z_{4}^{2}-\psi z_{1} z_{2}
\end{aligned}
$$

which describe non-compact Calabi-Yau spaces. The power $\ell$ of $x$ is determined by the Calabi-Yau condition, $\hat{c}=3$. We have also indicated the weights of the non-compact Calabi-Yau spaces, as well as the canonical hyperplane perturbation $\psi$ which deforms away from the Landau-Ginzburg point. Specifically, $\psi=\infty$ corresponds to the large complex structure limit, while the symmetric point $\psi=0$ gives the zero size limit of the del Pezzo 4-cycle. A natural coordinate on the moduli space $\mathcal{M}\left(\mathcal{B}_{n}\right)$ is given by $c=\psi^{-\ell}$, where $c=\left(e^{2 \pi i t_{S}}+\right.$ instanton corrections $)$ and $\ell=6,4,3,4$ for the three cases, respectively.

The point is that the period integrals over the corresponding holomorphic 3 -forms

$$
\tilde{\Pi}_{i}(c)=\int_{\Gamma_{i}} \Omega=\psi \int_{\Gamma_{i}} \frac{x d w d y d z_{1} d z_{2}}{W_{E_{n}}(c)}
$$

indeed reproduce the periods in (2.2) that govern our sub-monodromy problem. Here, $\Gamma_{i}$, $i=1, \ldots, 3$ denotes a basis of 3 -cycles on the surface $W_{E_{n}}=0$. These 3 -cycles are related via mirror symmetry to 0,2,4-cycles, where the 4-cycle is the del Pezzo surface itself. More 
concretely, the periods $\tilde{\Pi}_{i}$ are solutions of the following third-order Picard-Fuchs operators associated with the three-folds (4.2):

$$
\mathcal{L}^{(n)}=\mathcal{L}_{\text {ell }}^{(n)} \cdot \theta, \quad \theta \equiv c \frac{\partial}{\partial c}
$$

These form certain degenerate systems of generalized hypergeometric type ${ }_{3} F_{2}$, and are known [7] to govern the relevant periods of the del Pezzo surfaces. Above, the operators $\mathcal{L}_{\text {ell }}^{(n)}$, defined by

$$
\begin{array}{lll}
\mathcal{L}_{\text {ell }}^{(8)}=\theta^{2}-12 c(6 \theta+5)(6 \theta+1), & & \left(\frac{1}{6}, \frac{5}{6}, 1\right) \\
\mathcal{L}_{\text {ell }}^{(7)}=\theta^{2}-4 c(4 \theta+3)(4 \theta+1), & & \left(\frac{1}{4}, \frac{3}{4}, 1\right) \\
\mathcal{L}_{\text {ell }}^{(6)}=\theta^{2}-3 c(3 \theta+2)(3 \theta+1), & & \left(\frac{1}{3}, \frac{2}{3}, 1\right) \\
\mathcal{L}_{\text {ell }}^{(5)}=\theta^{2}-4 c(2 \theta+1)(2 \theta+1), & & \left(\frac{1}{2}, \frac{1}{2}, 1\right),
\end{array}
$$

are the Picard-Fuchs operators [28] of the following elliptic curves:

$$
\begin{aligned}
\mathbf{P}^{1,2,3}[6]: & P_{E_{8}}=y^{2}+w^{3}+z^{6}-\psi y w z, \\
\mathbf{P}^{1,1,2}[4]: & P_{E_{7}}=y^{2}+w^{4}+z^{4}-\psi y w z, \\
\mathbf{P}^{1,1,1}[3]: & P_{E_{6}}=y^{3}+w^{3}+z^{3}-\psi y w z, \\
\mathbf{P}^{1,1,1,1}[2,2]: & P_{E_{5}}^{1}=y^{2}+w^{2}-\psi v z, P_{E_{5}}^{2}=v^{2}+z^{2}-\psi y w .
\end{aligned}
$$

They define ordinary hypergeometric systems ${ }_{2} F_{1}$ of the types $(\alpha, \beta, \gamma)$ indicated on the r.h.s. of eq. (4.5). Therefore, the del Pezzo periods are related to the usual periods $\left(\varpi(c), \varpi_{D}(c)\right)=\psi \int d w / y$ of the tori (4.6) by

$$
\theta \cdot \tilde{\Pi}_{i} \equiv \theta \cdot\left(1, \tilde{\phi}(c), \tilde{\phi}_{D}(c)\right)=\left(0, \varpi(c), \varpi_{D}(c)\right)
$$

Interestingly one can treat the conifold singularity (4.1) in a similar way, by adding the hyperplane perturbation: $W_{C F}=\frac{1}{x}+y_{1}^{2}+y_{2}^{2}+y_{3}^{2}+y_{4}^{2}-\psi x y_{1} y_{2} y_{3} y_{4}$. This leads to a hypergeometric system of type $\left(\frac{1}{2}, \frac{1}{2}, 1\right)$, similar as for $E_{5}$, up to a subtle minus sign $c \rightarrow-c$ related to a half-integer shift of the B-field. 


\section{Topological properties, singularities and Gromov-Witten invariants}

The Calabi-Yau spaces (4.2) turn out to have quite interesting properties, suggesting a canonical structure also for the cases $\mathcal{B}_{n}, n<5$.

The topological properties can be summarized in terms of the triple self-intersections, the Euler number and an integral over the second Chern class, $c_{2}$ :

$$
\begin{aligned}
\int_{X} J \wedge J \wedge J & =n-9, \\
\int_{X} J \wedge c_{2} & =-12+2(n-9), \\
\int c_{3} & =\chi=-2 h\left(E_{n}\right),
\end{aligned}
$$

In particular, the Euler number is precisely twice the dual Coxeter number $h\left(E_{n}\right)$ of $E_{n}$ ! This agrees nicely with the physical expectation about the moduli space of the sixdimensional NCS, which involves the $U(1)$ gauge field and the hypermultiplets of the instanton moduli space (there is in addition one universal hypermultiplet which decouples together with gravity). Although we have derived these formulas from (4.2) only for the values $n=8,7,6,5$, it is clearly suggestive that they should apply also for the other values of $n$. In fact, the triple intersections agree with the self-intersections of $c_{1} \in H_{2}\left(\mathbf{B}_{n}, \mathbb{Z}\right)$ in $\mathbf{B}_{n}$ (and with the self-intersections of the elliptic curves (4.6) [29] as well). These facts further suggest that we really capture with the three-folds (4.2) the intrinsic information of the NCS associated with the del Pezzo surfaces.

There are three singular points with non-trivial monodromies in the moduli spaces $\mathcal{M}\left(\mathcal{B}_{n}\right)$, namely i) the large complex structure point $c=0$, ii) the point $c=\infty$, where the 4-cycle volume vanishes and iii) a conifold point at $1+d_{n} c=0$, where $d_{n}=432,64,27,16,-16$ for $n=8,7,6,5, C F$, respectively.

Let us comment on the nature of this conifold point. Firstly, it does not correspond to the conifold point that occurs in $\mathcal{B}_{n}^{\prime}$ at the flops, e.g. $t_{B}=0$ for $\mathcal{B}_{8}^{\prime}$. As indicated in Fig.1, the latter conifold point is not hit by the moduli space $\mathcal{M}\left(\mathcal{B}_{8}\right)$. Secondly, for the $\mathcal{B}_{n}$ theories, $1+d_{n} c=0$ requires negative values of $c$ and specifically a half-integer value of the $b$ field. Therefore this singularity is absent in the Lorentz invariant five and six dimensional theories. On the other hand, $c$ is positive at the conifold point of the "conifold" theory (4.1), as it should be for consistency. 
At $c=-d_{n}^{-1}$ there is a simple pole in the Yukawa coupling

$$
w(c)=\frac{1}{c^{3}\left(1+d_{n} c\right)},
$$

which, similar as in the compact case, obeys a linear differential equation,

$$
\left(3+4 d_{n} c\right) w(c)+c\left(1+d_{n} c\right) w^{\prime}(c)=0 .
$$

The Gromov-Witten invariants can be obtained from transforming (4.9) to special coordinates centered at the large complex structure point $c=0$. One finds the following instanton expansions for the Yukawa couplings $C_{\tilde{\phi} \tilde{\phi} \tilde{\phi}} \equiv \partial_{\tilde{\phi}}^{2} \tilde{\phi}_{D}$ :

$$
\begin{aligned}
& E_{8}:-1+252 \frac{1^{3} q^{1}}{1-q^{1}}-9252 \frac{2^{3} q^{2}}{1-q^{2}}+848628 \frac{3^{3} q^{3}}{1-q^{3}}-114265008 \frac{4^{3} q^{4}}{1-q^{4}}+\ldots, \\
& E_{7}:-2+56 \frac{1^{3} q^{1}}{1-q^{1}}-272 \frac{2^{3} q^{2}}{1-q^{2}}+3240 \frac{3^{3} q^{3}}{1-q^{3}}-58432 \frac{4^{3} q^{4}}{1-q^{4}}+1303840 \frac{5^{3} q^{5}}{1-q^{5}}+\ldots, \\
& E_{6}:-3+27 \frac{1^{3} q^{1}}{1-q^{1}}-54 \frac{2^{3} q^{2}}{1-q^{2}}+243 \frac{3^{3} q^{3}}{1-q^{3}}-1728 \frac{4^{3} q^{4}}{1-q^{4}}+15255 \frac{5^{3} q^{5}}{1-q^{5}}+\ldots, \\
& E_{5}:-4+16 \frac{1^{3} q^{1}}{1-q^{1}}-20 \frac{2^{3} q^{2}}{1-q^{2}}+48 \frac{3^{3} q^{3}}{1-q^{3}}-192 \frac{4^{3} q^{4}}{1-q^{4}}+960 \frac{5^{3} q^{5}}{1-q^{5}}+\ldots, \\
& C F: \quad 4+16 \frac{1^{3} q^{1}}{1-q^{1}}+16 \frac{2^{3} q^{2}}{1-q^{2}}+48 \frac{3^{3} q^{3}}{1-q^{3}}+192 \frac{4^{3} q^{4}}{1-q^{4}}+960 \frac{5^{3} q^{5}}{1-q^{5}}+\ldots,
\end{aligned}
$$

where $q \equiv e^{2 \pi i \tilde{\phi}}$. The instanton numbers agree with those calculated in a embedding Calabi-Yau for $n=8,7,6,5$ [7].

Interestingly, the expansion for the conifold theory (4.1) is related to that of $\mathcal{B}_{5}$ by a simple change of sign of $q$ in the Yukawa coupling, which corresponds to a half-integer shift of the $b$ field, as mentioned before. In particular, the non-compact Calabi-Yau space associated to $E_{5}$ and the conifold share the functional dependence of the periods on the modulus. The only difference is in the relative shift of the integral symplectic bases.

\section{Index of the singularities and elliptic curves}

As explained in [30], an important quantity characterizing the massless spectrum at the singularities is the singular behaviour of the topological amplitude $F_{1}^{t o p}$, defined in [31]. In the present case, the ansatz for $F_{1}^{t o p}$ is particularly simple:

$$
F_{1}^{t o p}=\log \left[c^{a}\left(1+d_{n} c\right)^{b} \frac{d c}{d t}\right]
$$

where $a, b$ are constants. These can be determined by comparison with the expression for $F_{1}^{t o p}$ in terms of world-sheet instantons of genus zero and ond:

$$
F_{1}^{\text {top }}=-\frac{2 \pi i}{12} t \int c_{2} \wedge J-\sum_{k}\left[2 n_{k}^{e} \log \left(\eta\left(q^{k}\right)\right)+\frac{1}{6} n_{k}^{r} \log \left(1-q^{k}\right)\right]+\text { const. }
$$

7 For the numbers $n_{k}^{e}$ of elliptic curves, see the Appendix. 
The massless spectrum at a singularity $c=c_{0}$ is then given by the coefficient $\beta$ of the logarithm, $F_{1}^{\text {top }} \sim-\beta / 6 \log \left(c-c_{0}\right)$, which counts the net number of massless vector hypermultiplets minus hypermultiplets, $\beta=n_{H}-n_{V}$.

At the conifold singularity, the above calculation yields $\beta=1$, as expected on general grounds. This singularity is therefore indeed due to one additional massless hypermultiplet.

More interesting is the behaviour at the point $c=\infty$, where the 4 -cycle vanishes. Taking carefully into account the contributions from the three factors in (4.11), namely a universal contribution $b=-\frac{1}{6}$ from the conifold discriminant, a factor $\left(\frac{7}{6}, \frac{5}{4}, \frac{4}{3}, \frac{3}{2}\right)$ from the determinant and $a=\left(-\frac{1}{6},-\frac{1}{3},-\frac{1}{2},-\frac{2}{3}\right)$, one finds

$$
\beta=(30,18,12,8)=h\left(E_{n}\right)
$$

for $\mathcal{B}_{n}, n=8,7,6,5$, respectively, where $h\left(E_{n}\right)$ is the dual Coxeter number of the global symmetry group $E_{n}$. The net massless spectrum at the vanishing 4-cycle singularity is therefore that of $h\left(E_{n}\right)$ extra massless hypermultiplets. This is in agreement with the change of hodge numbers proposed in [16] in the context of Calabi-Yau transitions.

In view of the bad convergence of the instanton expansion (4.10) at the conformal point $c=\infty$, this is quite remarkable. In particular, the number of massless BPS states arising from wrappings of vanishing 2-cycles is unbounded, indicating a breakdown of the description of the theory in terms of these degrees of freedom. The index $\beta$ sums up the contribution of the infinite number of terms to a finite result, indicating that the individual terms still make sense when appropriately regularized.

\section{Comparison with SYM theories with matter}

Note that the relations (4.7) are very reminiscent of Seiberg-Witten theory with extra matter [17]: derivatives of $\tilde{\phi} \sim a, \tilde{\phi}_{D} \sim a_{D}$ give torus periods. Moreover, the constant period, whose effects reflect Kaluza-Klein excitations in the present context (cf. (3.6)), corresponds to a bare mass. Indeed, the $E_{6,7,8}$ theories can formally be associated to the $S U(2)$ theories with $N_{f}=1,2,3$, respectively. That is, when tuning the mass parameters appropriately, one can check that the corresponding SW curves become equivalent (up to isogeny) to the $E_{6,7,8}$ tori (4.6); this is related to observations made in [32]. This implies that the effective $U(1)$ gauge couplings

$$
\tau(c) \equiv \frac{\partial^{2}}{\partial \tilde{\phi}^{2}} \mathcal{F}(\tilde{\phi})=\frac{\partial}{\partial \tilde{\phi}} \tilde{\phi}_{D}(\tilde{\phi})=\frac{\varpi_{D}(c)}{\varpi(c)},
$$


are, when viewed as functions of $c$, the same as the field theory couplings (e.g.., for small $c, \tau \sim \log (c))$.

However, the main difference from this viewpoint between the Yang-Mills theories and the $\mathcal{B}_{n}$ models is that the " $\theta$-operators", which map $\tilde{\phi}, \tilde{\phi}_{D}$ to the torus periods, are different. That is, $\theta=c \frac{\partial}{\partial c}$ is a logarithmic derivative and not an ordinary derivative. As a consequence, the couplings $\tau(\tilde{\phi})$ (and thus the prepotentials $\mathcal{F}(\tilde{\phi})$ ) as functions of $\tilde{\phi}$ are different as compared to ordinary field theories, and this implies that the theories $\mathcal{B}_{n}$ describe completely different physics.

Nevertheless the formal similarity suggests that the relevant geometry of the del Pezzo 4-cycle, and its moduli space $\mathcal{M}\left(\mathcal{B}_{n}\right)$, can be captured by SW curves given by the tori (4.6). For this to work, however, suitable meromorphic differentials $\lambda$ should exist on these curves that give rise to the periods $\tilde{\Pi}_{i}$. We will verify in the next section that such meromorphic forms can indeed be obtained directly from the 3 -forms, $\Omega$.

\section{Geometric reduction to elliptic curves}

We first consider the surface $W_{E_{8}}=0$ in weighted $\mathbf{P}^{4}$ for the $E_{8}$ Landau-Ginzburg potential (4.2). After re-defining $x$ and $y$ by some trivial phases, this surface can be written:

$$
y^{2}=w^{3}+z_{1}^{6}+z_{2}^{6}-\frac{1}{x^{6}}-\psi w x y z_{1} z_{2}
$$

Passing to the patch $x=1$, and integrating (4.3) with respect to $y$ around a contour about the surface $W_{E_{8}}=0$ yields:

$$
\int \Omega=\int \frac{\psi d w d z_{1} d z_{2}}{r}
$$

where

$$
r=\frac{\partial W_{E_{8}}}{\partial y}=2 \sqrt{w^{3}+z_{1}^{6}+z_{2}^{6}-1+\frac{1}{4}\left(\psi w z_{1} z_{2}\right)^{2}}
$$

We now wish to integrate $\Omega$ over some very particular 3-cycles in the surface (5.1). To exhibit these cycles we follow a simple variation of the approach of [19, 33, 23]. First we slice the surface by the simple quadrics, $w=\alpha z_{1}^{2}$, and use $\alpha$ and $z_{1}$ as new independent variables. Indeed we will consider $\alpha$ as parametrizing a $\mathbf{P}^{1}$ base, over which 2 -folds parametrized by $z_{1}$ and $z_{2}$ are fibered. We then identify 2-cycles in this 2-fold. 
Making the change of variables in $\Omega$, one obtains:

$$
\int \Omega=\int \frac{\psi d \alpha z_{1}^{2} d z_{1} d z_{2}}{2 \sqrt{\left(1+\alpha^{3}+\frac{1}{4}\left(\psi z_{2}\right)^{2} \alpha^{2}\right) z_{1}^{6}+\rho^{6}}}
$$

where

$$
\rho^{6}=z_{2}^{6}-1
$$

Considered as a function of $z_{1}, \Omega$ has six square root branch points. Integrate $\Omega$ along any contour, $\gamma_{1}$, that has $z_{1}$ large enough so as to encircle all these branch points. This contour can then be deformed out to infinity so as to give

$$
\frac{1}{2 \pi i} \oint_{\gamma_{1}} d z_{1} \Omega=\frac{\psi d \alpha d z_{2}}{2 \sqrt{\left(1+\alpha^{3}+\frac{1}{4}\left(\psi z_{2}\right)^{2} \alpha^{2}\right)}} .
$$

There are two natural integrals over $z_{2}$, and these correspond to integrals over two different types of 2-cycle:

(i) Integrate $z_{2}$ between any two zeroes of (5.5). This means that the $z_{1}$-contour then sweeps out a homology 2-sphere.

(ii) Integrate $z_{2}$ along any contour, $\gamma_{2}$, around the branch points of (5.6), and thus the $z_{1}$ and $z_{2}$ integration sweep out a 2-torus.

We consider the second possibility first: The integral over $z_{2}$ is elementary and yields

$$
\frac{1}{(2 \pi i)^{2}} \oint_{\gamma_{2}} d z_{2} \oint_{\gamma_{1}} d z_{1} \Omega=\frac{1}{2} \frac{d \alpha}{\alpha}
$$

Note that the factors of $\psi$ have cancelled. The last integral is then taken about $\alpha=0$ and gives

$$
\frac{2}{(2 \pi i)^{3}} \int \Omega=1
$$

which reproduces the constant period in $(2.2)$.

To perform the second integral one can perform the integration directly, but it is first instructive to note that

$$
\frac{d}{d \psi} \frac{\psi}{\sqrt{\left(1+\alpha^{3}+\frac{1}{4}\left(\psi z_{2}\right)^{2} \alpha^{2}\right)}}=\frac{d}{d z_{2}} \frac{z_{2}}{\sqrt{\left(1+\alpha^{3}+\frac{1}{4}\left(\psi z_{2}\right)^{2} \alpha^{2}\right)}}
$$


Hence if one differentiates the integral of interest with respect to $\psi$, then the $z_{2}$-integration becomes trivial: and the result is the difference, $\mathcal{I}_{k}-\mathcal{I}_{j}$, where

$$
\mathcal{I}_{k}=\frac{\zeta_{k}}{\sqrt{\left(1+\alpha^{3}+\frac{1}{4}\left(\psi \zeta_{k}\right)^{2} \alpha^{2}\right)}}
$$

and $\zeta_{k}=e^{\pi i k / 3}$. The remaining integration over $\alpha$ is a standard elliptic integral of the holomorphic differential, $d \alpha / \beta$, over the torus

$$
\beta^{2}=\alpha^{3}+\frac{1}{4}\left(\psi \zeta_{k}\right)^{2} \alpha^{2}+1
$$

which is equivalent to $P_{E_{8}}=0$ in (4.6).

There are two choices for this integral, the $a$-cycle or the $b$-cycle. To reconstruct the original integral of $\Omega$ over the corresponding 3-cycle, all we have to do is integrate these period integrals with respect to the modulus, $\psi$. In performing this integral with respect to $\psi$, the constant of integration is determined by the fact that for $\psi \rightarrow 0$, the integrand of the $z_{2}$-integral, $\psi\left(1+\alpha^{3}+\frac{1}{4}\left(\psi \zeta_{k}\right)^{2} \alpha^{2}\right)^{-1 / 2}$, vanishes linearly in $\psi$, while the range of the $z_{2}$-integration remains fixed (and finite). Hence these integrals vanish linearly in $\psi$ as $\psi \rightarrow 0$. (The first integral (5.8) does not vanish in this limit since the range of integration grows linearly with $\psi$.) Thus we see that the integral of $\Omega$ described in (i) reduces to evaluating the periods of a Seiberg-Witten differential associated with the torus (5.11).

The forgoing procedure is, of course, equivalent to integrating (5.6) with respect to $z_{2}$ between limits that are distinct values of $\zeta_{k}$. Doing this explicitly, one reduces the period integrals of $\Omega$ to differences of integrals of:

$$
\lambda_{k}=\frac{1}{2} \log \left[\frac{\sqrt{1+\alpha^{3}+\frac{1}{4}\left(\psi \zeta_{k}\right)^{2} \alpha^{2}}+\frac{1}{2} \psi \alpha \zeta_{k}}{\sqrt{1+\alpha^{3}+\frac{1}{4}\left(\psi \zeta_{k}\right)^{2} \alpha^{2}}-\frac{1}{2} \psi \alpha \zeta_{k}}\right] \frac{d \alpha}{\alpha}
$$

around the cycles of the corresponding tori (5.11). One can check that indeed $\theta \cdot \lambda_{k} \sim \frac{d \alpha}{\beta}$ up to exact pieces, which proves (4.7). Note also that $\lambda_{k}$ vanishes for $\psi=0$, which will prove important later.

We now briefly turn to the remaining cases. The computation for the surface $W_{E_{7}}=0$, or equivalently

$$
y^{2}=w^{4}+z_{1}^{4}+z_{2}^{4}-\frac{1}{x^{4}}-\psi w x y z_{1} z_{2}
$$


proceeds in an almost identical manner, except that slices the surface by hyperplanes, $w=\alpha z_{1}$, instead of quadrics. The integral over the 2 -torus as in (ii) above gives the result (5.8). Integrating over 2-spheres as in (i) above, leaves the differential:

$$
\lambda_{k}=\frac{1}{2} \log \left[\frac{\sqrt{1+\alpha^{4}+\frac{1}{4}\left(\psi \zeta_{k}\right)^{2} \alpha^{2}}+\frac{1}{2} \psi \alpha \zeta_{k}}{\sqrt{1+\alpha^{4}+\frac{1}{4}\left(\psi \zeta_{k}\right)^{2} \alpha^{2}}-\frac{1}{2} \psi \alpha \zeta_{k}}\right] \frac{d \alpha}{\alpha} .
$$

where $\zeta_{k}=e^{\pi i k / 2}$. This is to be integrated over the $a$-cycles and $b$-cycles of the associated torus

$$
\beta^{2}=\alpha^{4}+\frac{1}{4}\left(\psi \zeta_{k}\right)^{2} \alpha^{2}+1
$$

which is the same as $P_{E_{7}}=0$ in (4.6). Finally note that the the analysis for the surface $W_{E_{6}}=0$, or equivalently,

$$
y^{3}+w^{3}+z_{1}^{3}+z_{2}^{3}-\frac{1}{x^{3}}-\psi w x y z_{1} z_{2}=0
$$

is a little more complicated. One first makes a shift $w \rightarrow w-y$, followed by a shift $y \rightarrow y+\frac{1}{2} w$. The surface then becomes:

$$
\left(3 w+\psi x z_{1} z_{2}\right) y^{2}+\frac{1}{4} w^{3}+z_{1}^{3}-\frac{1}{4} \psi x w^{2} z_{1} z_{2}+z_{2}^{3}-\frac{1}{x^{3}}=0 .
$$

In the patch $x=1$, the 3 -form, $\Omega$, takes the form (5.2), with

$$
r=2 \sqrt{-\left(3 w+\psi z_{1} z_{2}\right)\left(\frac{1}{4} w^{3}+z_{1}^{3}-\frac{1}{4} \psi w^{2} z_{1} z_{2}+z_{2}^{3}-1\right)} .
$$

The computation now follows the usual pattern. Setting $w=\alpha z_{1}$, and changing variables from $w$ to $\alpha$, one can then integrate $z_{1}$ around a contour, $\gamma_{1}$, around both cuts in the $y$-plane. This leaves an integral of

$$
\frac{\psi d \alpha d z_{2}}{\sqrt{\left(3 \alpha+\psi z_{2}\right)\left(\psi z_{2} \alpha^{2}-\alpha^{3}-4\right)}}
$$

The denominator of the integrand is once again the square root of a quadratic in $z_{2}$. The Seiberg-Witten differential is:

$$
\lambda_{k}=\frac{1}{2} \log \left[\frac{\psi \zeta_{k} \alpha^{2}+\left(\alpha^{3}-2\right)+\alpha \sqrt{\left(3 \alpha+\psi \zeta_{k}\right)\left(\psi \zeta_{k} \alpha^{2}-\alpha^{3}-4\right)}}{\psi \zeta_{k} \alpha^{2}+\left(\alpha^{3}-2\right)-\alpha \sqrt{\left(3 \alpha+\psi \zeta_{k}\right)\left(\psi \zeta_{k} \alpha^{2}-\alpha^{3}-4\right)}}\right] \frac{d \alpha}{\alpha}
$$

on the Riemann surface

$$
\beta^{2}=\left(3 \alpha+\psi \zeta_{k}\right)\left(\psi \zeta_{k} \alpha^{2}-\alpha^{3}-4\right)
$$


Upon simply substituting $\beta=2\left(3(w+y)+\psi z_{1} \zeta_{k}\right)(y-w) / z_{1}^{2}$ and $\alpha=(w+y) / z_{1}$, this curve is easily seen to be equivalent to the torus $P_{E_{6}}=0$ in (4.6).

We did not try to perform an analogous computation for the $E_{5}$ potential in (4.2), but have no doubt that it would work out in a similar way.

One should note that there are some fundamental differences between the foregoing calculations and the usual calculation of Seiberg and Witten. Apart from the vanishing of (5.12) at $\psi=0$, which is a regular point of the moduli space, it should be noted that the differential (5.12) is not single valued. Logarithms certainly arise in some of the standard forms of $\lambda$, but usually these logarithms can be removed by suitable integrations by parts. Put another way, the branches of the logarithm play no role because the shift of $2 \pi i$ associated with crossing the cuts merely adds the (cohomologically trivial) derivative of a meromorphic function to $\lambda$. In the foregoing, this ambiguity in the logarithm adds multiples of $2 \pi i(d \alpha / \alpha)$ to $\lambda_{k}$, and this has residues at $\alpha=0, \infty$.

This result is somewhat new from the one-dimensional perspective of the SeibergWitten differential, but it is easily understood form the perspective of the 3 -fold. There is an obvious ambiguity in the integrations of type (i) between the zeroes of (5.5): the path can wind around the set of all branch points of the integrand of (5.6), exactly as in (ii).

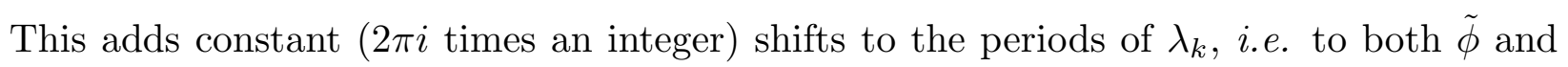
$\tilde{\phi}_{D}$.

However, from the one-dimensional perspective, while there is only one torus, there are infinitely many Seiberg-Witten differentials, $\lambda_{(n)}$, which differ only by multiples of $d \alpha / \alpha$ so that $\lambda_{(n)}$ has residue $2 \pi i n$ at $\alpha=0$. If one now tries to use the Seiberg-Witten differential as a metric, as in [19], then one must take infinitely many copies, indexed by $n$, of the basic torus, with a metric obtained from $\lambda_{(n)}$ on the $n^{\text {th }}$ copy. This foliation of the basic torus is sewn together at the logarithmic branch cuts of $\lambda_{(n)}$. For the differential (5.12), these cuts are located at $\alpha=e^{2 \pi i / 3}$. BPS geodesics can thus find their way between sheets by cycling around these branch points, and thus the counting of BPS states [19] will be very different as compared to ordinary $N=2$ Yang-Mills theories. 


\section{Periods, prepotentials and monodromies from the $E_{8}$ torus}

In order to obtain explicit expressions for the geometric del Pezzo periods, $\tilde{\Pi}_{i}$, we can now simply make use of the hypergeometric systems (4.5) related to the SW tori (4.6). The correct integral symplectic linear combinations of the solutions can be simply determined by matching the asymptotic expansions with the period integrals over the differentials $\lambda$ of the previous section.

To be specific, we will consider here only the model $\mathcal{B}_{8}$; the other theories can be treated in an analogous way. For $\lambda$ is as in (5.12), we find, in terms of the inverse variable $u \equiv-1 /(432 c)$ and a standard homology basis $\gamma_{a, b}$ on the $E_{8}$ torus, the following expressions for the periods:

$$
\begin{aligned}
\tilde{\phi}(u) & =\frac{3}{\pi^{2}} \int_{\gamma_{a}} \lambda=\left(\int \varpi(u) \frac{d u}{u}\right)+\delta \\
\tilde{\phi}_{D}(u) & =\frac{3}{\pi^{2}} \int_{\gamma_{b}} \lambda=\left(\int \varpi_{D}(u) \frac{d u}{u}\right)+\delta_{D} .
\end{aligned}
$$

Here, $\delta, \delta_{D}$ are integration constants, and

$$
\begin{aligned}
\varpi_{D}(u) & =\frac{3^{1 / 4}}{4 \pi^{3 / 2} i}\left(\xi F_{0}(u)+\frac{1}{\xi} F_{1}(u)\right) \\
\varpi(u) & =\frac{3^{1 / 4}}{4 \pi^{3 / 2} i}\left(\rho \xi F_{0}(u)+\frac{1}{\rho \xi} F_{1}(u)\right),
\end{aligned}
$$

with $\xi \equiv-\frac{i 3^{1 / 4}}{2^{2 / 3} \pi^{3 / 2}} \Gamma(1 / 3)^{3}, \rho \equiv e^{2 \pi i / 3}$ and

$$
F_{0}(u)=u^{1 / 6}{ }_{2} F_{1}\left(\frac{1}{6}, \frac{1}{6}, \frac{1}{3} ; u\right), \quad F_{1}(u)=u^{5 / 6}{ }_{2} F_{1}\left(\frac{5}{6}, \frac{5}{6}, \frac{5}{3} ; u\right) .
$$

The hypergeometric system is known to have singularities at $u=0,1, \infty$. With the help of well-known formulas for the analytic continuation of ${ }_{2} F_{1}$, we can easily find expansions of $\tilde{\phi}(u), \tilde{\phi}_{D}(u)$ in local patches near these singularities. However, when comparing different patches, we must be careful with the integration constants $\delta, \delta_{D}$ in (6.1). For expansions around $u=0$ these constants must be zero, since we know from the vanishing of $\lambda$ (5.12) for $u=0$ that both periods $\tilde{\phi}$ and $\tilde{\phi}_{D}$ vanish there. From this, or from the explicit expression for $\lambda$, we can match the integration constants in other patches.

Explicitly, we find the following local expansions near the singularities:

1) Near $u=0$, which is the point of intersection with the theory discussed in [8,15]:

$$
(2 \pi i) \tilde{\phi}_{D}(u)=-\frac{3^{5 / 4}}{\sqrt{\pi}} u^{1 / 6}\left[\xi\left(1+\frac{1}{84} u+\mathcal{O}\left(u^{2}\right)\right)+\frac{1}{\xi} u^{2 / 3}\left(\frac{1}{5}+\frac{5}{132} u+\mathcal{O}\left(u^{2}\right)\right)\right]
$$


and $\tilde{\phi}(u)=\left.\tilde{\phi}_{D}(u)\right|_{\xi \rightarrow \rho \xi}$. The vanishing of both $\tilde{\phi}$ and $\tilde{\phi}_{D}$, as well as the absence of a logarithm, reflects conformal invariance of the theory at $u=0$. This is similar to the $S U(2)$ theories with $N_{f}=1,2,3$ [24, 32]. The prepotential $\mathcal{F}_{0}(\tilde{\phi})=\frac{1}{2} \tau_{F P} \tilde{\phi}^{2}-\frac{i}{10 \sqrt{3} \xi^{6}} \tilde{\phi}^{6}+\mathcal{O}\left(\tilde{\phi}^{10}\right)$ is thus polynomial in $\tilde{\phi}$, and for the $U(1)$ coupling constant (4.13) at the origin we have: $\tau_{F P}=\rho^{2}$. This is, as expected, a fixed point of the modular group (this value is consistent with $u(\tau)=\frac{1}{864}(J(\tau)+\sqrt{J(\tau)(J(\tau)-1728)})$, from which we find for the other fixed point of the modular group: $u(\tau=i)=2)$.

2) Near $u=1$ :

$$
\begin{gathered}
(2 \pi i) \tilde{\phi}(u)=\text { const. }-\frac{1}{2 \pi}\left[\left\{(u-1)(1+\log [432])+\mathcal{O}\left((u-1)^{2}\right)\right\}-\right. \\
\left.\left(i-i \tilde{\phi}_{D}(u)\right) \log [u-1]\right] \\
(2 \pi i) \tilde{\phi}_{D}(u)=2 \pi i+i(u-1)-\frac{31}{72} i(u-1)^{2}+\mathcal{O}\left((u-1)^{3}\right)
\end{gathered}
$$

This means that at $u=1$ there is a conifold singularity that is analogous to the SW monopole singularity. It corresponds to a massless BPS state with quantum numbers $\left(n_{0}, n_{2}, m_{2}\right)=(-1,0,1)$.

3) Near $u=\infty$ :

For these expansions we go back to the original variable, $c=-1 /(432 u)$. From the analytic continuation we have the following integration constants:

$$
\delta=\frac{1}{2}-\frac{1}{2 \pi i} \log [432], \quad \delta_{D}=\frac{13}{24}+\frac{1}{8 \pi^{2}} \log [432]^{2},
$$

and thus:

$$
\begin{aligned}
(2 \pi i) \tilde{\phi}(c)= & \log [c]-60 c+6930 c^{2}+\mathcal{O}\left(c^{3}\right) \\
(2 \pi i) \tilde{\phi}_{D}(c)= & \frac{5}{6} i \pi-\left(\left(30+126 \frac{i}{\pi}\right) c+\mathcal{O}\left(c^{2}\right)\right)+ \\
& \left(\frac{1}{2}-30 \frac{i}{\pi} c+\mathcal{O}\left(c^{2}\right)\right) \log [c]-\frac{1}{4 \pi i} \log [c]^{2} .
\end{aligned}
$$

The SW period $\tilde{\phi}_{D}(c)$ indeed reproduces the three-fold period $-\Pi_{3}$, given in (3.3) and (A.2) below, when expressed in terms of $t_{S} \equiv \tilde{\phi}(u)$. Consequently, up to a constant, the prepotential near $u=\infty$ is:

$$
\mathcal{F}_{\infty}(\tilde{\phi})=\frac{1}{6} \tilde{\phi}^{3}+\frac{1}{4} \tilde{\phi}^{2}-\frac{5}{12} \tilde{\phi}+\frac{1}{8 \pi^{3} i} \sum_{k=1}^{\infty} n_{k}^{r} L i_{3}\left(e^{2 \pi i k \tilde{\phi}}\right)
$$

where the instanton coefficients $n_{k}^{r}=\{252,-9252, .$.$\} are as in (4.10), and L i_{3}$ is the trilogarithm [34]. This rigid prepotential is obviously quite different from the usual SW 
theory prepotentials, $\mathcal{F}_{\infty}(a) \sim a^{2} \log a^{2}+\mathcal{O}(1 / a)$ for large $a$, and also from that of a higherdimensional field theory including the effects of Kaluza-Klein excitations. We stress again that (6.7) reflects the behaviour of the rigid sub-moduli problem at the large complex structure point of the threefold. This is different than in usual field theory embeddings in string theory, where the "point at infinity" of the field theory does not coincide with the "point at infinity" of the string theory.

From the above local expansions we find the following monodromy matrices:

$$
M_{0}=\left(\begin{array}{ccc}
1 & 0 & 0 \\
0 & 0 & -1 \\
0 & 1 & 1
\end{array}\right), \quad M_{1}=\left(\begin{array}{ccc}
1 & 0 & 0 \\
-1 & 1 & 1 \\
0 & 0 & 1
\end{array}\right), \quad M_{\infty}=\left(\begin{array}{ccc}
1 & 0 & 0 \\
-1 & 1 & 0 \\
0 & 1 & 1
\end{array}\right)
$$

which act on $\tilde{\Pi}=\left(1, \tilde{\phi}, \tilde{\phi}_{D}\right)$ and which represent the quantum symmetries of the theory. They obey $M_{1} \cdot M_{0}=M_{\infty}, M_{0}{ }^{6}=\mathbb{1}$, and we can easily identify the modular group $S L(2, \mathbb{Z})$ in the lower right block, i.e.., $M_{0} \sim T^{-1} S, M_{1} \sim T S^{-1} T$ and $M_{\infty} \sim T$. From these generators we can build pure shifts: $\tilde{\phi} \rightarrow \tilde{\phi} \pm 1$ or $\tilde{\phi}_{D} \rightarrow \tilde{\phi}_{D} \pm 1$. These can be subtracted from the above generators, and thus the full duality group is given by a semi-direct product of $S L(2, \mathbb{Z})$ with $\mathbb{Z} \times \mathbb{Z}$.

\section{Discussion}

In this paper we have investigated del Pezzo singularities of Calabi-Yau three-folds in $N=2$ supersymmetric type IIA string compactifications. We have isolated a onedimensional moduli space, $\mathcal{M}\left(\mathcal{B}_{n}\right)$, which intersects the large complex structure point of the three-folds. This moduli space corresponds to a closed sub-monodromy problem, and should therefore be associated with a consistent sub-theory of the full string compactification.

The question arises as to what the structure of this consistent sub-theory is, and what precise physical meaning the moduli space has. This is like asking what the physical meaning of the moduli space of a Seiberg-Witten curve is, without a priori knowing the underlying Yang-Mills theory. In other words, we need to ask what the physical system is whose moduli space is the moduli space of a del Pezzo surface.

Clearly, although the theory has six non-compact dimensions (since we used a noncompact threefold to model it), it does not describe a six-dimensional Lorentz invariant theory. Moreover the effective description in terms of special geometry is an essentially four dimensional concept. However, the situation should be compared to the effective theory 
obtained in the limit of a large base $\mathbf{P}^{1}$ in a K3 fibered Calabi-Yau manifold: although the global geometry does not allow to recover precisely the six-dimensional space-time theory of the pure $K 3$ compactification, one does recover the precise moduli space of the $K 3$ compactification, that is, the singularities, monodromies and gauge coupling dependence. This is because the $\mathbf{P}^{1}$ becomes locally flat and the local properties of the theory become indistinguishable from that of the truly six-dimensional theory.

From the discussion in sections 2 and 3 we know that the coordinate $c$ of the moduli space describes essentially the string tension of the six-dimensional non-critical $E_{n}$ string. In particular there are no relevant geometrical parameters to vary. It is therefore suggestive to interpret this moduli space primarily as the moduli space of the six-dimensional string theory itself, and not of a particular compactification. Specifically, its singularities should be related to the properties of the string theory itself, and not just to an interplay with the geometry of the compactification. The behaviour at $c=\infty$, where the string tension vanishes, is clearly consistent with this picture. The large complex structure point, $c=0$, is stringy either: the behaviour of the theory at this point is characterized in terms of the type II A string world-sheet instantons expansion. These instantons are supported by the same rational curves which give also rise to the BPS states of the NCS relevant at the $c=\infty$ singularity. On the other hand, the third singular point, the conifold singularity, is slightly different in that it needs a non-vanishing value of the $b$ field in the directions in which Lorentz invariance is broken, and would therefore be absent in a Lorentz invariant theory.

In order to gain a better understanding of the structure of the theory, it would be interesting to repeat the conformal field theory analysis of [27] in the present context. It would also be important to understand the precise counting of the BPS states, as well as their underlying algebraic structure. For the counting, the SW formulation involving the peculiar differentials $\lambda$ might be very useful. As for the algebraic structure, recall that the homology of the del Pezzo surfaces is $\operatorname{dim} H_{*}\left(\mathbf{B}_{n}, \mathbb{Z}\right)=\left(b_{0}=1, b_{2}=n+1, b_{4}=1\right)$, which leads to a Lorentzian period lattice of type $\Gamma_{n+2,2} . H_{2}$ forms a sub-lattice of type $\Gamma_{n+1,1}$, which is related to the period lattice [35] of the relevant simple elliptic singularity in (4.6) (note that we considered in this paper only a one-dimensional sub-lattice of $H_{2}$ ). We expect the BPS states to form a generalized Kac-Moody algebra [34 associated with the period lattice, and the prepotential $\mathcal{F}$ and the topological free energy $\mathcal{F}_{1}$ to be associated with the corresponding automorphic forms.

Finally, it would also be instructive to investigate the precise relationship of the $N=2$ supersymmetric theories discussed in this paper with the $E_{n}$ superconformal fixed points of 
[23]. These are based on the simple singularities of type $E_{n}$, while the theories discussed in the present paper are related to the elliptic singularities of type $\hat{E}_{n}$. It is well-known from the adjacency diagrams of singularity theory [29] that the $E_{n}$ singularities can be obtained by deformations of the $\hat{E}_{n}$ singularities. Both types of theories have superconformal points in their moduli spaces, and we suspect these to belong to different universality classes. Very crudely speaking, the $\hat{E}_{n}$ superconformal fixed point might be a stringy, "affine" version of the $E_{n}$ superconformal fixed point.

We believe it would be important to shed more light on these issues, and intend to report on them in the future. As a remark, note also in this context that we can treat the conifold singularity (4.1) in a very similar way as we did for the del Pezzo singularities. Since this singularity is much more generic than the del Pezzo singularities, we might gain considerable understanding of non-perturbative string theory by studying this theory in more detail.

\section{Acknowledgements}

We would like to thank J. Minahan, D. Nemeschansky, S. Theisen and C. Vafa for valuable discussions. N.W. is supported in part by funds provided by the DOE under grant number DE-FG03-84ER-40168. 


\section{Appendix A. Prepotential and periods of the three-fold $\mathrm{X}_{\mathrm{F}_{1}}$}

The elliptically fibered three-fold $\mathbf{X}_{\mathbf{F}_{1}}$ with base $\mathbf{F}_{1}$ has hodge numbers $h^{1,1}=$ $3, h^{1,2}=243$. Its description in terms of toric geometry and its phases has been discussed in much detail in [16, 36, 37,7].

The four-dimensional $N=2$ vector moduli space is described by the holomorphic prepotential of special geometry $\mathcal{F}\left(t_{i}\right)$. For $\mathbf{X}_{\mathbf{F}_{1}}$ it takes the following asymptotic form in the two phases, Phase $I$ and Phase $I I$ :

$$
\begin{aligned}
\mathcal{F}_{I}\left(t_{i}\right)= & -\frac{1}{2} t_{F}^{2} t_{E}-t_{F} t_{B} t_{E}-\frac{3}{2} t_{F} t_{E}^{2}-t_{B} t_{E}^{2}-\frac{4}{3} t_{E}^{3} \\
& +\frac{1}{2} t_{F} t_{E}+\frac{3}{2} t_{F}+t_{B}+\frac{23}{6} t_{E}-c_{0}, \\
\mathcal{F}_{I I}\left(t_{i}\right)= & -\frac{1}{2} t_{F}^{\prime 2} t_{B}^{\prime}-\frac{1}{2} t_{F}^{\prime 2} t_{E}^{\prime}-\frac{3}{2} t_{F}^{\prime} t_{B}^{\prime 2}-3 t_{F}^{\prime} t_{B}^{\prime} t_{E}^{\prime}-\frac{3}{2} t_{F}^{\prime} t_{E}^{\prime 2}-\frac{3}{2} t_{B}^{\prime 3} \\
& -\frac{9}{2} t_{B}^{\prime} t_{E}^{\prime}-\frac{9}{2} t_{B}^{\prime} t_{E}^{\prime 2}-\frac{4}{3} t_{E}^{\prime 3}+\frac{1}{2} t_{F}^{\prime} t_{B}^{\prime}+\frac{1}{2} t_{F}^{\prime} t_{E}^{\prime}+\frac{1}{4} t_{B}^{\prime 2} \\
& +\frac{1}{2} t_{B}^{\prime} t_{E}^{\prime}+\frac{3}{2} t_{F}^{\prime}+\frac{17}{4} t_{B}^{\prime}+\frac{23}{6} t_{E}^{\prime}-c_{0},
\end{aligned}
$$

where $c_{0}=-i \zeta(3) / 2(2 \pi)^{3} \chi$. The primed coordinates in Phase $I I$ are related to those in Phase $I$ by $t_{B}^{\prime}=-t_{B}, t_{F}^{\prime}=t_{F}+t_{B}, t_{E}^{\prime}=t_{S}=t_{E}+t_{B}$. The volume of the 4-cycle is $t_{S}$, while the volume of the 2-cycle shrinking at the flop is $t_{B}$.

The period vector $\Pi$ has entries $\left(\mathcal{F}_{0}, \mathcal{F}_{i}, 1, t_{i}\right)$ in a basis with canonical symplectic metric. Applying an integral symplectic transformation puts it into the following form:

$$
\Pi_{I I}=\left(\begin{array}{c}
\frac{1}{2} t_{F}^{\prime 2} t_{E}+\frac{3}{2} t_{F}^{\prime} t_{E}^{2}-\frac{1}{6} t_{S}^{3}+\frac{3}{2} t_{E}^{3}+\frac{3}{2} t_{F}^{\prime}-\frac{5}{12} t_{S}+\frac{17}{4} t_{E}-2 c_{0} \\
-t_{F}^{\prime} t_{E}-\frac{3}{2} t_{E}^{2}+\frac{1}{2} t_{E}+\frac{3}{2} \\
\frac{1}{2} t_{S}^{2}-\frac{1}{2} t_{S}-\frac{5}{12} \\
-\frac{1}{2} t_{F}^{\prime 2}-3 t_{F}^{\prime} t_{E}-\frac{9}{2} t_{E}^{2}+\frac{1}{2} t_{F}^{\prime}+\frac{1}{2} t_{E}+\frac{17}{4} \\
1 \\
t_{F}^{\prime} \\
t_{S} \\
t_{E}
\end{array}\right)
$$

The periods $\left(1, t_{S}, \Pi_{3}\right)$ depend then (classically) only on the Kähler coordinate $t_{S}$ and define our sub-monodromy problem. Moreover, $t_{E}$ is the additional period treated as a background field in the $\mathcal{B}_{n}^{\prime}$ theory. 


\begin{tabular}{|c|c|c|c|c|c|c|c|c|c|}
\hline & $d$ & 1 & 2 & 3 & 4 & 5 & 6 & 7 & 8 \\
\hline$n$ & & & & & & & & & \\
\hline 8 & & -2 & 762 & -246788 & 76413073 & -23436186174 & 7209650619780 & -2232321201926990 & 696061505044554010 \\
\hline 7 & & 0 & 3 & -224 & 12042 & -574896 & 26127574 & -1163157616 & 51336812456 \\
\hline 6 & & 0 & 0 & -4 & 135 & -3132 & 62976 & -1187892 & 21731112 \\
\hline 5 & & 0 & 0 & 0 & 5 & -96 & 1280 & -14816 & 160784 \\
\hline 0 & & 0 & 0 & -10 & 231 & -4452 & 80958 & -1438086 & 25301064 \\
\hline
\end{tabular}

Table 1: Number of genus one curves of degree $d$ for $\mathcal{B}_{n}, n=8,7,6,5,0$.

For comparison we have also given the number of genus one curves for $\mathcal{B}_{0}$ in table 1 , which have been determined in 38. 


\section{References}

[1] E. Witten, Some Comments on String Dynamics, hep-th/9507121.

[2] N. Seiberg and E. Witten, Nucl. Phys. B471 (1996) 121

[3] O. J. Ganor and A. Hanany, Nucl. Phys. B474 (1996) 122

[4] E. Witten, Nucl. Phys. B471 (1996) 195

[5] O. Ganor, Six-dimensional Tensionless Strings in the Large N Limit, hep-th/9605201; Compactification of Tensionless String Theories., hep-th/9607092.

[6] O. J. Ganor, Nucl. Phys. $\underline{B 479}$ (1996) 197

[7] A. Klemm, P. Mayr and C. Vafa, BPS states of exceptional non-critical strings, hepth/9607139.

[8] O. Ganor, Toroidal Compactification of Heterotic 6D Non-Critical Strings Down to Four Dimensions, hep-th/9608109.

[9] E. Witten, Physical Interpretation Of Certain Strong Coupling Singularities, hepth/9609159

[10] P. Mayr, Mirror Symmetry, $N=1$ Superpotentials and Tensionless Strings on CalabiYau Four-Folds, hep-th/9610162

[11] J. Distler and A. Hanany, (0,2) Noncritical Strings in Six Dimensions, hep-th/9611104.

[12] N. Seiberg, Phys. Lett. $\underline{B 388}$ (1996) 753

[13] D. R. Morrison and N. Seiberg, Extremal Transitions and Five-Dimensional Supersymmetric Field Theories, hep-th/9609070

[14] M. Douglas, S. Katz and C. Vafa, Small Instantons, Del Pezzo Surfaces and Type I' theory, hep-th/9609071

[15] O.J. Ganor, D.R. Morrison and N. Seiberg, Branes, Calabi-Yau Spaces, and Toroidal Compactification of the N=1 Six-Dimensional $E_{8}$ Theory, hep-th/9610251

[16] D. R. Morrison and C. Vafa, Nucl. Phys. $\underline{B 473}$ (1996) 74, Nucl. Phys. $\underline{B 476}$ (1996) 437

[17] N. Seiberg and E. Witten, Nucl. Phys. B426 (1994) 19; Nucl. Phys. B431 (1994) 484

[18] S. Kachru, A. Klemm, W. Lerche, P. Mayr and C. Vafa, Nucl. Phys. B459 (1996) 537

[19] A. Klemm, W. Lerche, P. Mayr, C. Vafa and N.P. Warner, Nucl. Phys. B477 (1996) 746

[20] M. Günaydin, G. Sierra and P.K. Townsend, Nucl. Phys. B242 (1994) 244; Nucl. Phys. $\underline{B 253}(1985) 573$ 
[21] I. Antoniadis, S. Ferrara and T.R. Taylor, Nucl. Phys. B460 (1996) 489

[22] A. Ceresole, R. D'Auria, S. Ferrara and A. Van Proeyen, Nucl. Phys. B444 (1995) 92

[23] J. Minahan and D. Nemeschansky, An N=2 Superconformal Fixed Point with $E_{6}$ Global Symmetry, hep-th/9608047; Superconformal Fixed Points with $E_{n}$ Global Symmetry, hep-th/9610076

[24] P. C. Argyres, M. R. Plesser, N. Seiberg and E. Witten, Nucl. Phys. B461 (1996) 71

[25] C. Vafa and D. Goshal, Nucl. Phys. B453 (1995) 121.

[26] S. Mukhi and C. Vafa, Nucl. Phys. $\underline{B 407}$ (1993) 667

[27] H. Ooguri and C. Vafa, Nucl. Phys. $\underline{B 463}$ (1996) 55

[28] B. Lian and S.-T. Yau, Comm. Math. Phys. 176 (1996) 163.

[29] K. Saito, Einfach-Elliptische Singularitäten, Inv. Math. 23 (1974) 289.

[30] C. Vafa, Nucl. Phys. B477 (1995) 262

[31] M. Bershadsky, S. Ceccotti, H. Ooguri and C. Vafa (App. by S. Katz), Nucl. Phys. $\underline{B 405}$ (1993) 279, Comm. Math. Phys. 165 (1994) 311

[32] T. Masuda and H. Suzuki, Periods and Prepotential of $N=2 S U(2)$ Supersymmetric Yang-Mills Theory with Massive Hypermultiplets, hep-th/9609066.

[33] W. Lerche and N.P. Warner, Exceptional SW Geometry from ALE Fibrations, hepth/9608183.

[34] J. Harvey and G. Moore, Nucl. Phys. $\underline{B 463}$ (1996) 315; On the Algebras of BPS States, hep-th/9609017.

[35] See e.g., V. Arnold, A. Gusein-Zade and A. Varchenko, Singularities of Differentiable Maps I, II, Birkhäuser 1985.

[36] P. Candelas and A. Font, Duality Between Webs of Heterotic and Type II Vacua, hep-th/9603170

[37] J. Louis, J. Sonnenschein, S. Theisen and S. Yankielowicz, Nucl. Phys. B4480 (1996) 185

[38] P. Candelas, X. De la Ossa, A. Font, S. Katz, and D. Morrison, Nucl. Phys. $\underline{B 429}$ (1994) 626 